\title{
Decomposition of Heartbeat Time Series: Scaling Analysis of the Sign Sequence
}

\author{
Y Ashkenazy ${ }^{1}$, PCh Ivanov ${ }^{1,2}$, S Havlin ${ }^{3}$, C-K Peng ${ }^{2}$, Y Yamamoto ${ }^{4}$, AL Goldberger ${ }^{2}$, HE Stanley ${ }^{1}$ \\ ${ }^{1}$ Center for Polymer Studies and Department of Physics, Boston University, Boston, Massachusetts \\ 02215, USA \\ ${ }^{2}$ Harvard Medical School, Beth Israel Deaconess Medical Center, Boston, Massachusetts 02215, USA \\ ${ }^{3}$ Dept. of Physics and Gonda Goldschmied Center, Bar-Ilan University, Ramat-Gan, Israel \\ ${ }^{4}$ Tokyo University, Graduate School of Education, Education Physiol. Lab., Bunkyo Ku, 7-3-1 Hongo, \\ Tokyo 1130033, Japan
}

\begin{abstract}
The cardiac interbeat $(R R)$ increment time series can be decomposed into two sub-sequences: a magnitude series and a sign series. We show that the sign sequence, a simple binary representation of the original $R R$ series, retains fundamental scaling properties of the original series, is robust with respect to outliers, and may provide useful information about neuroautonomic control mechanisms.
\end{abstract}

\section{Introduction}

Many biological and physical systems exhibit complex dynamics characterized by long-range correlation properties (scaling laws) [1, 2, 3]. For example, apparently noisy normal cardiac interbeat $(R R)$ interval time series obey scaling laws and the RR increment series shows long-range anticorrelations [4]. Moreover, these scaling exponents may have diagnostic and prognostic utility [5, 6].

In a previous report [7] we showed that two components of the heart interbeat interval increment series, namely the magnitude ("volatility") and sign, can be of help in understanding the underlying dynamics which relate to correlation properties in the original heartbeat time series (Figs. 1 and 2). The heartbeat increment magnitude series was shown to reveal some nonlinear aspects of the original heartbeat increment series. On the other hand, the heartbeat increment sign series mainly reflects the linear properties of the heartbeat increment series. The correlation properties of the sign series may also be of use in separating healthy patients from heart-failure patients. Surprisingly, the sign series yields equivalent, and in some cases better separation of these two groups than the original heartbeat interval time series.

Here we focus in further detail on the sign sequence of the $R R$ increment series. We show that sign series is robust in handling complex signals which include spikes.

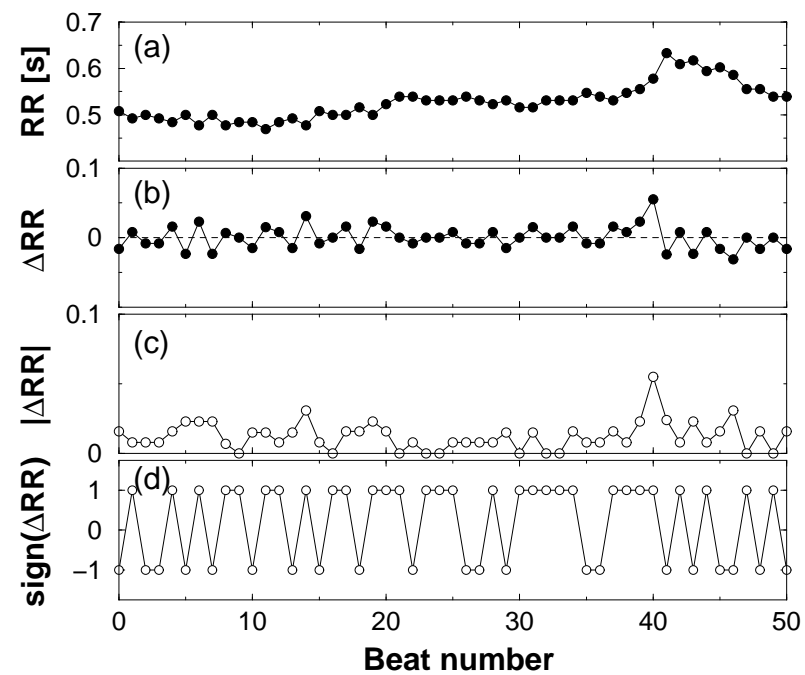

Figure 1. An illustration of the magnitude/sign decomposition. (a) The $R R$ time series (healthy subject) versus beat number for 50 beats. (b) The increment, $\Delta R R$ of the $R R$ time series shown in (a). (c) Magnitude of the increments of the successive heartbeat intervals of the series shown in (a). Patches of less "volatile" increments with small magnitude (beat number 20-35) are followed by patches of more volatile increments with large magnitude (beat number 35-50), suggesting that there is correlation in the magnitude time series. (b) The sign sequence, of the $R R$ series shown in (a). The positive sign $(+1)$ represents a positive increment, while the negative sign $(-1)$ represents a negative increment in the $R R$ series of interbeat intervals. The complex alternation between +1 and -1 is consistent with the finding [7] that there is multi-scale anticorrelation in the sign time series.

In addition we demonstrate that $\beta$-blockade in healthy young adult subjects causes an increase in the short range anticorrelated behavior of the $R R$ increment time series 


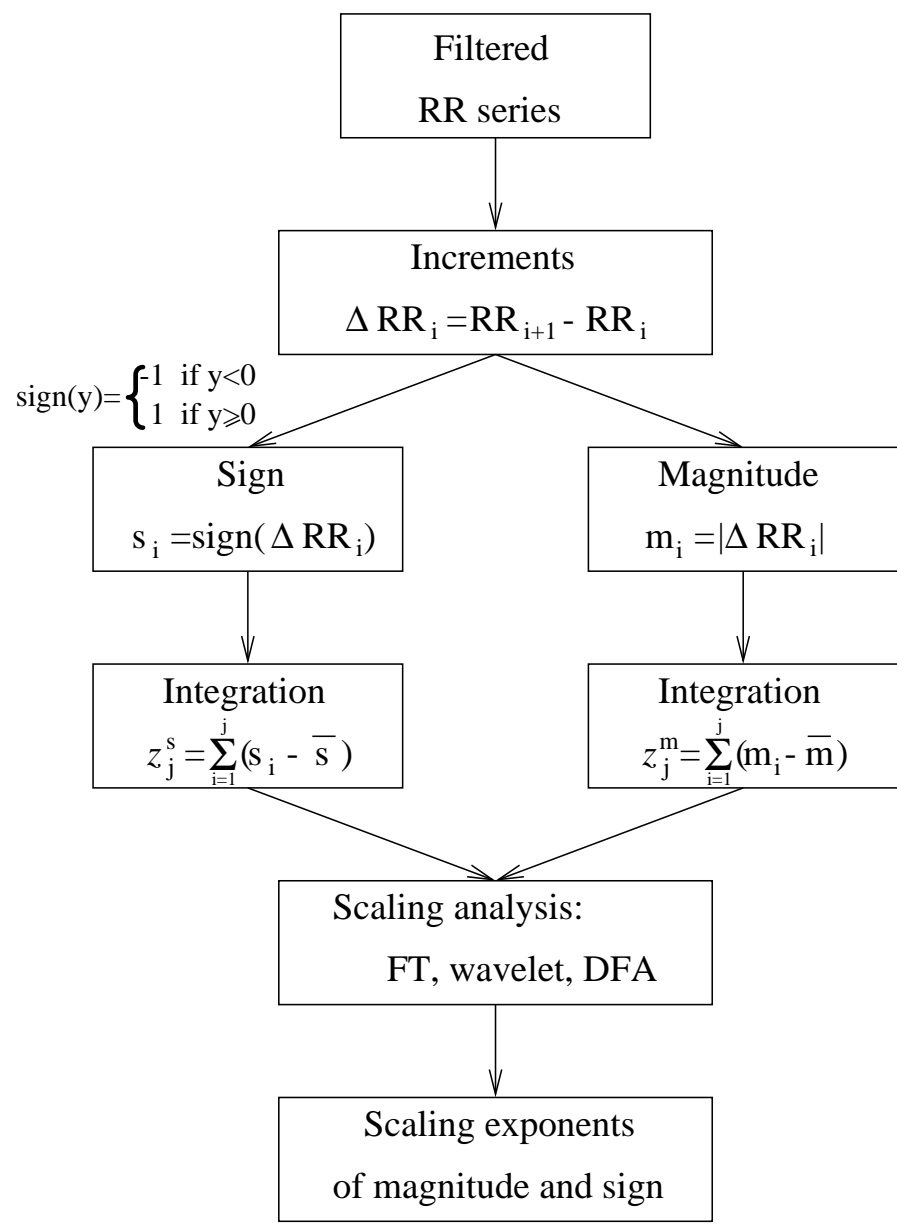

Figure 2. A schematic representation of the magnitude/sign decomposition of the $R R$ time series. FT: Fourier transform; DFA: detrended fluctuation analysis.

and its component sign series. Our results, therefore, suggest that the simple sign series can mimic the shortrange correlation properties of the original $R R$ series and that it may have advantages when considering noisy data with many outliers.

\section{Method}

The time series of the fluctuations in heartbeat intervals can be "decomposed" into two different time series. This procedure allows for separate analysis of the time sub-series formed by the magnitude and the sign of the increments in the time intervals between successive heartbeats (Figs. 1] and (2).

Given a filtered $R R_{i}$ series we generate the increment $R R$ series, namely, $\Delta R R_{i}=R R_{i+1}-R R_{i}$. Then, the increment series is decomposed into two sub-series: the magnitude series $m_{i}=\left|\Delta R R_{i}\right|$, and sign series
$\left.s_{i}=\operatorname{sign}\left(\Delta R R_{i}\right)\right]^{m}$. Then we integrate the magnitude and sign sub-series. To avoid artificial trends, prior to the integration step we subtract from the magnitude and sign series their averages. Then, we use the detrended fluctuation analysis (DFA) method [\$] to calculate the root mean square fluctuation function, $F(n)$, where $n$ is the window scale.

The DFA method excludes nonstationarities which arise because of trends that are not necessarily related to the cardiac dynamics. The $1^{\text {st }}$ order DFA method excludes constant trends that exist in the original series; the $2^{\text {nd }}$ order DFA excludes linear trends that appear in the data, and higher order of DFA excludes higher order of polynomial trends. In our case, namely, the $R R$ time series, we found that the $2^{\text {nd }}$ order of DFA is adequatef

The final step, is to calculate the scaling exponents of the integrated magnitude and sign series. The scaling exponent, $\alpha$, is the exponent which quantifies the growth of the root mean square fluctuations, $F(n) \propto n^{\alpha}$. These steps are schematically summarized in Fig 2 .

We note that other methods, such as Fourier transform and wavelet transform [8], can be applied to calculate the scaling exponents. In these cases one can avoid the integration step of the magnitude and sign decomposition (see Fig. 27) and measure the scaling exponents directly from the magnitude and sign series. However, we use the DFA method because of its ability to exclude trends and its simplicity.

\section{Examples}

\subsection{Surrogate noise}

In real life examples, like the heartbeat $R R$ time series, outliers due to noise, missing beats or extrasystoles that appear in the recorded signal might alter the scaling exponent calculation. However, by definition, the sign series does not contain any outliers, and thus preserves the correct scaling properties despite the spikes existing in the original signal.

To illustrate this point, we generate complex series with intrinsic long-range correlations [9] (we chose scaling exponent, $\alpha=0.3$, similar to the long-range scaling exponent that observed in some $R R$ increment time series). Then, we replace some of the data points with spikes. We then compare the root mean square fluctuation function

\footnotetext{
${ }^{1}$ The sign of zero is considered as 1 .

${ }^{2}$ The $F(n)$ curves are used to approximate the scaling exponents that characterize the long range correlation of a given series. The $q^{\text {th }}$ order of DFA method can measure accurately series with scaling exponents, $\alpha$, that are in the range of $1 / 2 \leq \alpha \leq q+1 / 2$. In the present case the scaling exponents do not exceed the value of 1.8 and thus the $2^{\text {nd }}$ order of DFA is adequate. Moreover, we have applied higher order DFA to our data and find similar values for the computed scaling exponents.
} 


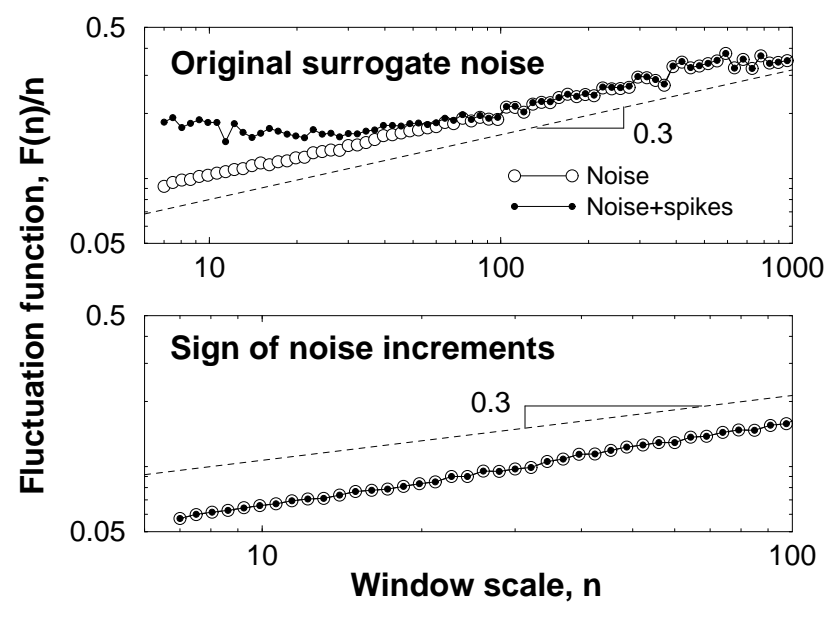

Figure 3. We generate a long-range correlated series (16384 data points) with scaling exponent of $\alpha=0.3$ for the increment series with and without spikes. Then, we calculate the root mean square fluctuation function $F(n)$ both of the original surrogate series $(\circ)$ and of the surrogate series with added spikes $(\bullet)$. The upper panel shows the results of the original long-range correlated noise; the noise+spike fluctuation function is very much different than the original noise fluctuation function. The integrated sign series shows identical results for both series. The dashed line indicates the slope (scaling exponent) of 0.3 .

$F(n)$ of the two series, namely, the original long-range correlated series and the series which contains spikes. We repeat this procedure for the integrated sign series obtained from the two surrogate series.

In Fig. 3 we show the results for series of 16384 data points. The spike size is 10 times the standard deviation of the original surrogate series. We replaced just 5 data points with spikes. It is clear from the figure that the short-range correlation properties of the original surrogate noise are lost due to the appearance of these spikes, while the integrated sign series preserves the scaling properties of the original sign series.

\section{2. $\beta$-Blockade}

Healthy subjects under the influence of $\beta$-blockade may exhibit increased high frequency $R R$ behavior [10]. This prominent high frequency behavior is consistent with the strong alternations and large variability observed in the data as compared to placebo control (Fig. 円). The alternations of the heart interbeat increments can readily visualized, in the sign series. These alternations suggest stronger anticorrelated behavior (for both the $R R$ series and the sign series) under the influence of the $\beta$-blocking drug.

Our correlation analysis indeed shows stronger anticorrelated behavior after $\beta$-blockade for $R R$ series as well as for the sign series (Fig. 5). These results suggest that interaction between the sympathetic and the parasympathetic systems is reflected by the sign of the heartbeat increments.

\section{Summary}

We study one of the most basic representations of cardiac interbeat increment dynamics - the sign series. We show that it is robust to outliers which may appear in the heartbeat time series. Moreover, we show that the sign series reflects changes in short-range neuroautonomic effects. The sign series may add additional information and may aid in cardiac diagnosis and prognosis in certain conditions.

\section{Acknowledgments}

Partial support was provided by the NIH/National Center for Research Resources (P41 RR13622) the G. Harold and Leila Y. Mathers Charitable Foundation, the Fetzer Foundation, and the Israel-USA Binational Science Foundation.

\section{References}

[1] Shlesinger MF. Fractal time and $1 / f$ noise in complex systems. Ann NY Acad Sci 1987;504:214-228.

[2] Vicsek T. Fractal Growth Phenomena. Second edition. Singapore: World Scientific, 1993.

[3] Takayasu H. Fractals in the Physical Sciences. Manchester: Manchester Univ. Press, 1997.

[4] Kobayashi M, Musha T. $1 / f$ fluctuation of heartbeat period. IEEE Trans Biomed Eng 1982;29:456-457.

[5] Peng CK, Havlin S, Stanley HE, Goldberger AL. Quantification of scaling exponents and crossover phenomena in nonstationary time series. Chaos 1995; 5:82-87. Also www.physionet.org/tutorials/fmnc/.

[6] Huikuri HV, Makikallio TH, Peng CK, Goldberger AL, Hintze U, Moller M ;Fractal correlation properties of R-R interval dynamics and mortality in patients with depressed left ventricular function after an acute myocardial infarction. Circulation 2000;101:47-53.

[7] Ashkenazy Y, Ivanov PC, Havlin S, Peng CK, Goldberger AL, Stanley HE. Magnitude and sign correlations in heartbeat fluctuations ;Submitted for publication; http://xxx.lanl.gov/abs/cond-mat/0005365.

[8] Ivanov PC, Amaral LAN, Goldberger AL, Havlin S, Rosenblum MG, Struzik ZR, Stanley HE. Multifractality in human heartbeat dynamics. Nature 1999;399:461-465.

[9] Makse H, Havlin S, Schwartz M, H.E. S. Method for generating long-range correlations for large systems. Phys Rev E 1996;53:5445-5449.

[10] Yamamoto Y, Hughson RL. On the fractal nature of heart rate variability in humans. Am J Physiol 1994;266:R40R49. 
$\beta$ - Blockade
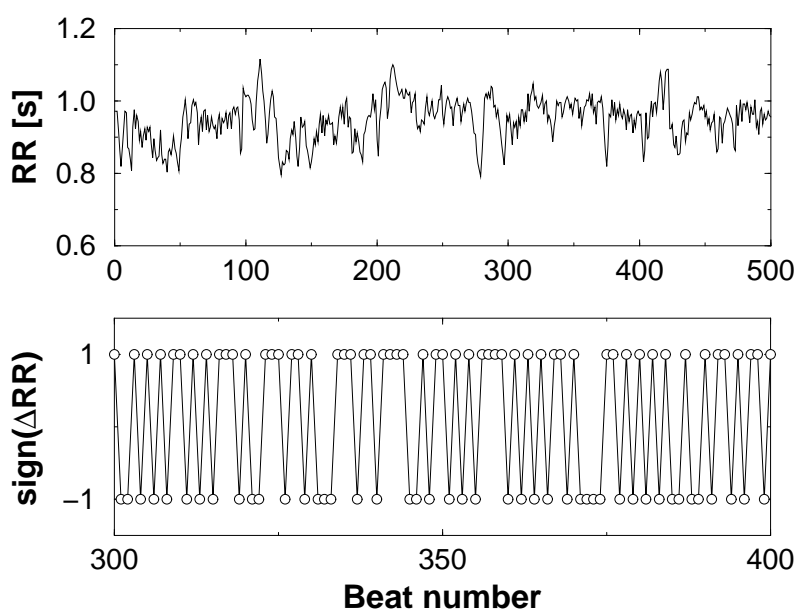

Placebo
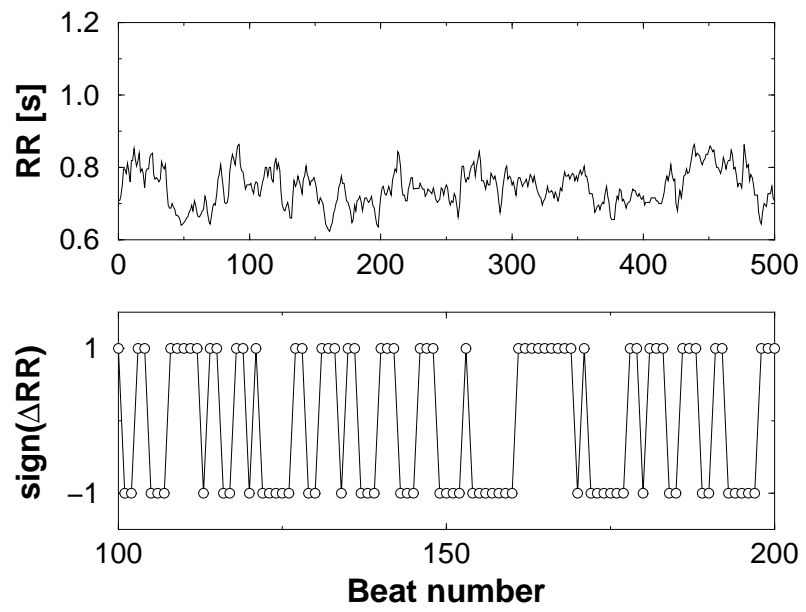

Figure 4. Examples of heartbeat interval time series after administration of a long-acting $\beta$-blocking drug, metoprolol tartrate, $120 \mathrm{mg}$ (left panels) and under normal conditions (placebo, right panels) in a young adult healthy subject. The $\beta$-blockade time series and its component sign series show more alternations which suggest stronger anticorrelated behavior compared with placebo conditions as confirmed by quantitative scaling analysis. See Fig. 5 .
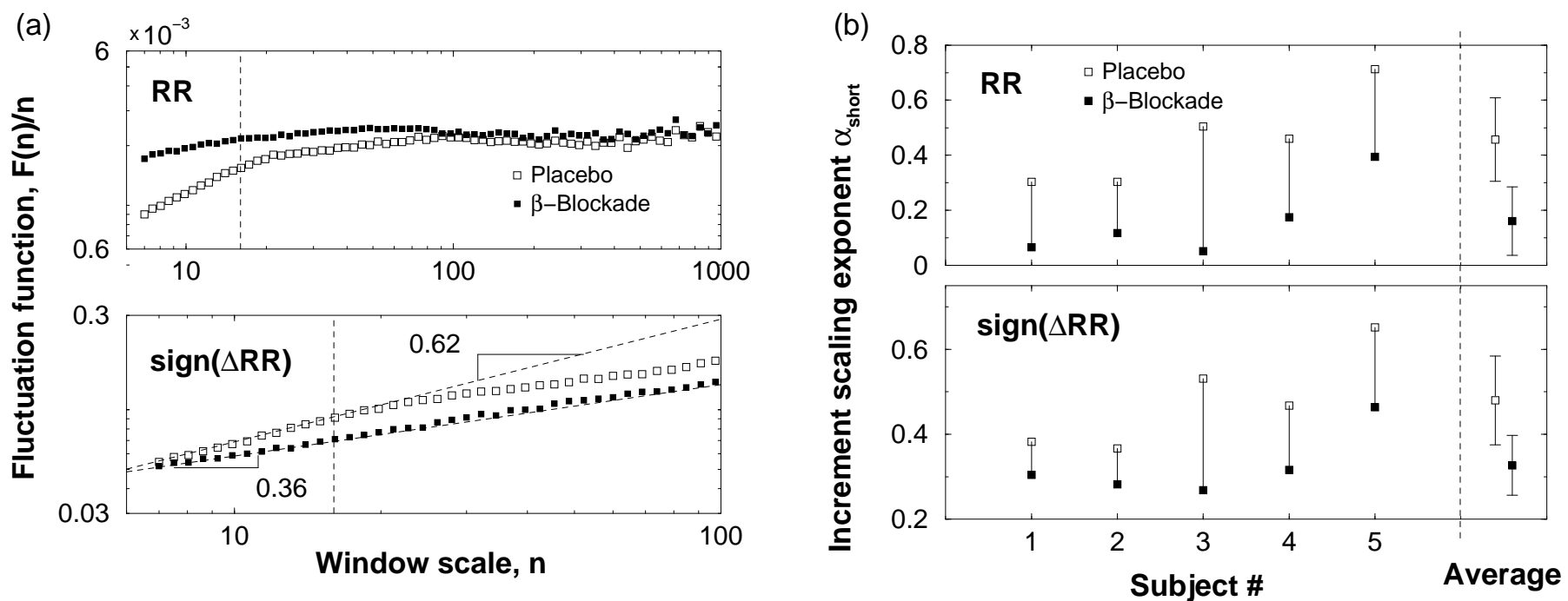

Figure 5. (a) Effect of $\beta$-blockade in healthy young subjects. The scaling of the original $R R$ time series changes at short time scales and instead of an apparent crossover at time scale $n \approx 16$ beats (indicated by the dashed vertical line), we have a single scaling exponent. For the sign series we observe the same changes as in the original data. (b) A summary of 14 records, 30000 data points each, from 5 subjects. The subjects were recorded either with placebo or $\beta$-blockade therapy (subjects 2 and 5 have 2 records each both for $\beta$-blockade and placebo; the average value is given.) The figure shows a systematic decrease of $\alpha_{\text {short }}$, the short range scaling exponent ( $7<n<16$ beats) both for the original $R R$ series as well as for the sign series. This finding suggests that the short-range scaling exponent relates to the interaction between the sympathetic and the parasympathetic components of the neuroautonomic nervous system. The error bars indicate the mean \pm 1 standard deviation. 